\title{
Neutron Skin Effects in Mirror Energy Differences: The Case of ${ }^{23} \mathrm{Mg}^{23} \mathrm{Na}$
}

A. Boso, ${ }^{1,2, *}$ S. M. Lenzi, ${ }^{1,2}$ F. Recchia, ${ }^{1,2}$ J. Bonnard, ${ }^{2,3}$ A. P. Zuker, ${ }^{1,4}$ S. Aydin, ${ }^{5}$ M. A. Bentley, ${ }^{6}$ B. Cederwall, ${ }^{7}$ E. Clement, ${ }^{8}$ G. de France, ${ }^{8}$ A. Di Nitto, ${ }^{9,10}$ A. Dijon, ${ }^{8}$ M. Doncel, ${ }^{7}$ F. Ghazi-Moradi, ${ }^{7}$ A. Gadea, ${ }^{11}$ A. Gottardo, ${ }^{12}$ T. Henry, ${ }^{6}$ T. Hüyük, ${ }^{11}$ G. Jaworski, ${ }^{13}$ P. R. John, ${ }^{1,2, \dagger}$ K. Juhász, ${ }^{14}$ I. Kuti, ${ }^{14}$ B. Melon, ${ }^{15}$ D. Mengoni, ${ }^{1,2}$ C. Michelagnoli, ${ }^{1,2}$ V. Modamio, ${ }^{12}$ D. R. Napoli, ${ }^{12}$ B. M. Nyakó, ${ }^{14}$ J. Nyberg, ${ }^{16}$ M. Palacz, ${ }^{13}$ J. Timár, ${ }^{14}$ and J. J. Valiente-Dobón ${ }^{12}$

${ }^{1}$ Dipartimento di Fisica e Astronomia, Università degli Studi di Padova, I-35131 Padova, Italy

${ }^{2}$ INFN, Sezione di Padova, I-35131 Padova, Italy

${ }^{3}$ Institut de Physique Nucléaire, IN2P3-CNRS, Université Paris-Sud, Université Paris-Saclay, F-91405 Orsay, France

${ }^{4}$ Universitè de Strasbourg and IPHC, F-67000 Strasbourg, France

${ }^{5}$ Aksaray Universitesi, Department of Physics, TR-68100 Aksaray, Turkey

${ }^{6}$ University of York, Department of Physics, YO10 5DD York, United Kingdom

${ }^{7}$ Department of Physics, Royal Institute of Technology, SE-10691 Stockholm, Sweden

${ }^{8}$ GANIL, CEA/DRF-CNRS/IN2P3, F-14076 Caen, France

${ }^{9}$ Dipartimento di Fisica and INFN, Sezione di Napoli, I-80126 Napoli, Italy

${ }^{10}$ Johannes Gutenberg-Universität Mainz, 55099 Mainz, Germany

${ }^{11}$ IFIC, CSIC-Universitat de València, E-46980 Valencia, Spain

${ }^{12}$ INFN, Laboratori Nazionali di Legnaro, I-35020 Legnaro (Padova), Italy

${ }^{13}$ Heavy Ion Laboratory, Warsaw University, 02-093 Warszawa, Poland

${ }^{14}$ Institute of Nuclear Research (ATOMKI) of Hung. Acad. Sciences, H-4001 Debrecen, Hungary

${ }^{15}$ Dipartimento di Fisica and INFN, Sezione di Firenze, I-50019 Firenze, Italy

${ }^{16}$ Department of Physics and Astronomy, Uppsala University, SE-75120 Uppsala, Sweden

(Received 13 April 2018; published 19 July 2018)

\begin{abstract}
Energy differences between analogue states in the $T=1 / 2{ }^{23} \mathrm{Mg}-{ }^{23} \mathrm{Na}$ mirror nuclei have been measured along the rotational yrast bands. This allows us to search for effects arising from isospin-symmetrybreaking interactions (ISB) and/or shape changes. Data are interpreted in the shell model framework following the method successfully applied to nuclei in the $f_{7 / 2}$ shell. It is shown that the introduction of a schematic ISB interaction of the same type of that used in the $f_{7 / 2}$ shell is needed to reproduce the data. An alternative novel description, applied here for the first time, relies on the use of an effective interaction deduced from a realistic charge-dependent chiral nucleon-nucleon potential. This analysis provides two important results: (i) The mirror energy differences give direct insight into the nuclear skin; (ii) the skin changes along the rotational bands are strongly correlated with the difference between the neutron and proton occupations of the $s_{1 / 2}$ "halo" orbit.
\end{abstract}

DOI: 10.1103/PhysRevLett.121.032502

Radii of atomic nuclei are strongly influenced by the nucleons arrangement in the valence orbitals. In particular, it has recently been shown [1] that low- $l$ orbitals extend much farther than their partners in a main shell. Therefore, the variation of the occupation numbers of neutron and protons in low- $l$ orbits as a function of the angular momentum of the nucleus has an important impact on the evolution of the neutron and proton radii and of their difference, i.e., the neutron skin.

Direct measurements of the nucleon distribution of shortlived excited states are not feasible. However, the neutron skin affects structural properties as, for example, the difference in excitation energy of analog states in nuclei with exchanged numbers of protons and neutrons, the socalled mirror energy differences (MED). The study of the MED becomes thus a powerful indirect method to access the nuclear radii and skin.
MED have been exploited extensively as a tool to understand the evolution of the nuclear structure properties as a function of the angular momentum and to test the isospin symmetry of the strong interaction in the nuclear medium [2,3]. Systematic studies of the behavior of the MED as a function of the angular momentum have been performed in the $f_{7 / 2}$ shell up to rather high spin. A theoretical shell-model description, using an effective charge-independent nuclear interaction plus Coulomb matrix elements, has shown to reproduce very accurately the available data, provided two correction terms are included: (i) a monopole Coulomb contribution that takes into account the change of radius of the nuclei with increasing angular momentum and excitation energy [4], and (ii) a schematic isospin symmetry-breaking (ISB) term [2]. This latter term is not compatible with the ISB of effective interactions deduced from realistic 
charge-dependent nucleon-nucleon potentials. Indeed, it has been shown that they fail to reproduce the MED data [5].

The success of the studies of MED in the $f_{7 / 2}$ shell relies on the good amount of data. On the other hand, the experimental information in other shells is scarce and limited to few states, as the neutron-deficient partners become rapidly unbound. In the low $s d$ shell, however, the development of deformation offers the possibility to study the MED along several yrast states in rotational bands and to establish if the radial and ISB correction terms, deduced for the $f_{7 / 2}$ shell, constitute a general and robust feature.

Here we report new experimental results for MED up to rather high spin for the mirror pair ${ }^{23} \mathrm{Mg}^{23} \mathrm{Na}$, which presents ground-state rotational-like bands. This allows us to test the validity of the theoretical description used in the $f_{7 / 2}$ shell and to apply for the first time an alternative approach based on a realistic chiral nucleon-nucleon interaction. Prior to this study, $\gamma$-ray spectroscopy information on the ${ }^{23} \mathrm{Na}$ nucleus extended up to spin $17 / 2^{+}$at 11.1 MeV, has been reported by Jenkins et al. [6]. In the same work, the nucleus ${ }^{23} \mathrm{Mg}$ was observed up to $J^{\pi}=$ $13 / 2^{+}$at $7.1 \mathrm{MeV}$. Early studies based on neutron and charged-particle spectroscopy tentatively assigned levels up to $J^{\pi}=21 / 2^{+}$in ${ }^{23} \mathrm{Na}$ [7] and reported states up to $14 \mathrm{MeV}$ excitation energy in ${ }^{23} \mathrm{Mg}$ [8], but spin and parity of states above $11 / 2^{+}$were only tentative.

Excited states in mirror nuclei of mass $A=23$ were populated via the ${ }^{12} \mathrm{C}\left({ }^{16} \mathrm{O}, \alpha n\right){ }^{23} \mathrm{Mg}$ and ${ }^{12} \mathrm{C}\left({ }^{16} \mathrm{O}, \alpha p\right){ }^{23} \mathrm{Na}$ fusion-evaporation reactions. The ${ }^{16} \mathrm{O}$ beam was provided by the CIME cyclotron of GANIL with two different energies of 60 and $70 \mathrm{MeV}$. The target consisted of a $500 \mu \mathrm{g} / \mathrm{cm}^{2}$ self-supported ${ }^{12} \mathrm{C}$ foil. The channels of interest were selected via prompt coincidences with the particles evaporated in the decay of the ${ }^{28} \mathrm{Si}$ compound nucleus. Charged particles were detected by the $4 \pi$ array DIAMANT, composed of 80 CsI scintillators [9]. Neutrons were identified by the Neutron Wall [10], consisting in 50 BC501A scintillators. The $\gamma$ rays emitted in the deexcitation of the evaporation residues were detected by the EXOGAM array [11] composed of ten Comptonsuppressed HPGe segmented clover detectors: seven placed at $90^{\circ}$ and three at $135^{\circ}$. Events were sorted off-line into $E_{\gamma}-E_{\gamma}$ matrices in coincidence with the evaporated particles. To improve the Doppler correction, an eventby-event kinematical reconstruction of the velocity vector of the recoiling nuclei was performed. In Fig. 1 the spectra corresponding to the $A=23$ mirror nuclei are shown.

The transitions up to the highest spin states previously known are clearly observed, corresponding to the $17 / 2^{+} \rightarrow$ $15 / 2^{+} 2034 \mathrm{keV}$ line in ${ }^{23} \mathrm{Na}$ and the $13 / 2^{+} \rightarrow 9 / 2^{+}$ $3480 \mathrm{keV}$ peak in ${ }^{23} \mathrm{Mg}$. In addition, four new transitions have been observed in ${ }^{23} \mathrm{Mg}$, evidenced in Fig. 1. The $E_{\gamma}-E_{\gamma}$ analysis and the striking analogy with the ${ }^{23} \mathrm{Na}$ spectrum allow us to firmly assign them to the decay of the

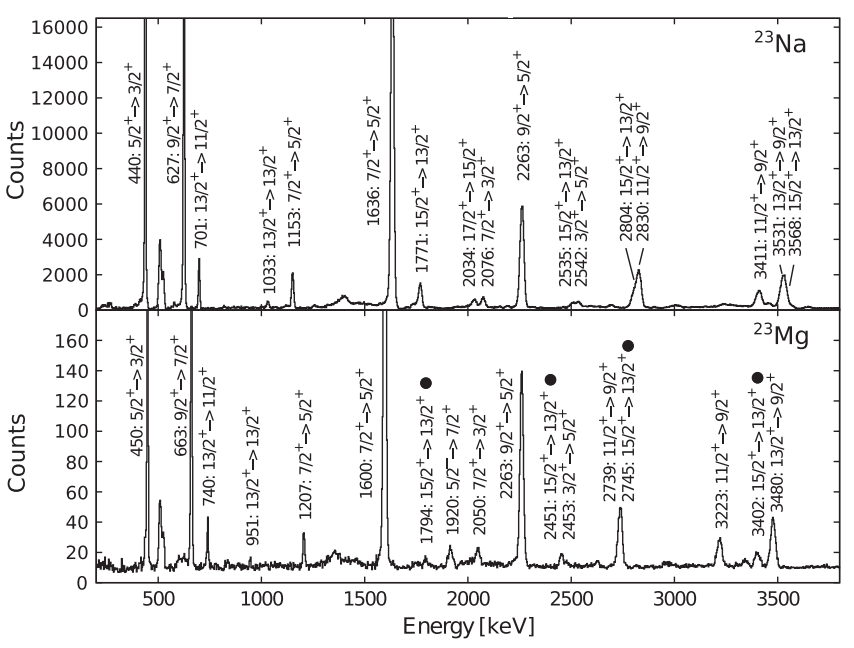

FIG. 1. Gamma-ray spectra of ${ }^{23} \mathrm{Na}$ and ${ }^{23} \mathrm{Mg}$ in coincidence with one $\alpha$ particle and one proton and one $\alpha$ particle and one neutron, respectively. A sum of gates on the 440 and $627 \mathrm{keV}$ and on the 450 and $663 \mathrm{keV}$ transitions in ${ }^{23} \mathrm{Na}$ and ${ }^{23} \mathrm{Mg}$, respectively, has been applied to the $E_{\gamma}-E_{\gamma}$ matrix. The newly identified transitions in ${ }^{23} \mathrm{Mg}$ are marked with dots.

two $J^{\pi}=15 / 2^{+}$states at 8937 and $9594 \mathrm{keV}$ excitation energy, respectively. The resulting level schemes for the positive-parity states are reported in Fig. 2, where the width of the arrows is proportional to the relative intensity of each transition, properly corrected for the efficiency of the setup. The excitation energies of the two signatures of the yrast sequence of both nuclei show the characteristic rotational pattern compatible with an intrinsic deformed shape. The MED between the rotational analogue states up to $J^{\pi}=15 / 2^{+}$, are reported in Fig. 3 .

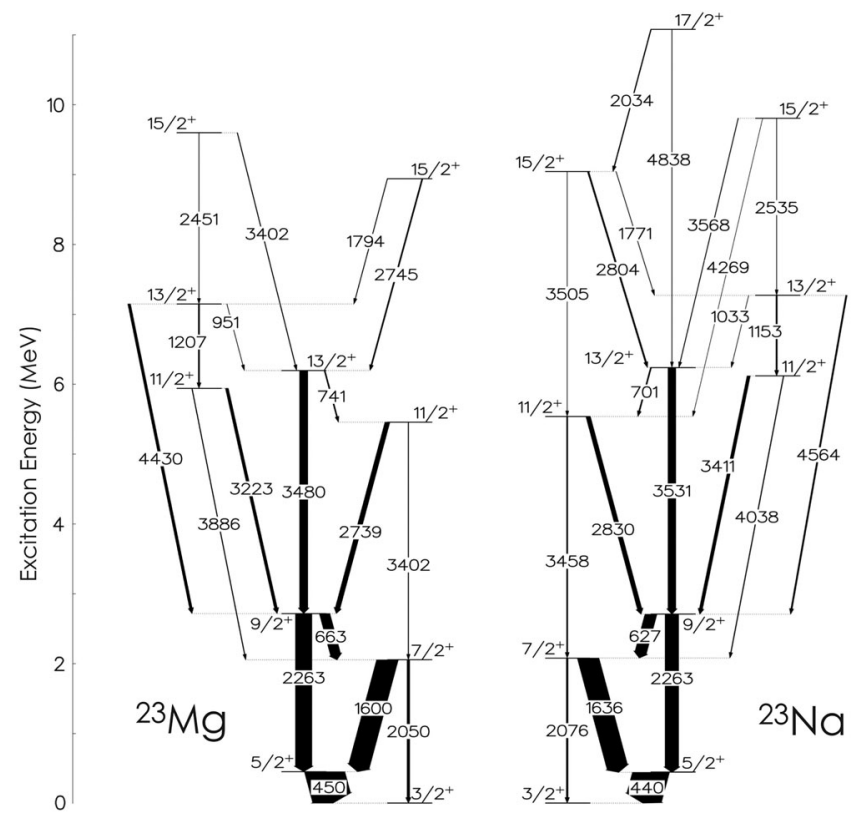

FIG. 2. Level schemes of ${ }^{23} \mathrm{Na}$ and ${ }^{23} \mathrm{Mg}$ deduced from particle$\gamma-\gamma$ coincidences. 
To theoretically analyze the MED we follow, as a first approach, the method successfully adopted in the $f_{7 / 2}$ shell and reported in Refs. [2,3]. The MED as a function of the angular momentum $J$ are obtained, in first order perturbation theory, as the sum of the Coulomb multipole $V_{\mathrm{CM}}$ and monopole $V_{C m}$ terms and an additional ISB term, called $V_{B}$ :

$$
\operatorname{MED}(J)=\Delta\left\langle V_{\mathrm{CM}}\right\rangle_{J}+\Delta\left\langle V_{C m}\right\rangle_{J}+\Delta\left\langle V_{B}\right\rangle_{J},
$$

where $\Delta$ indicates the difference between the analogue states with angular momentum $J$ of the two mirror nuclei. The isospin-symmetric nuclear effective interaction USD [12] has been used in the shell model calculations. The multipole Coulomb matrix elements of $V_{\mathrm{CM}}$ have been obtained in the harmonic-oscillator basis and the singleparticle energies have been corrected to include the monopole effects arising from the electromagnetic spinorbit interaction [13] and the orbital term [14]. Two additional terms, the monopole Coulomb $V_{C m}$ and the isovector ISB $V_{B}$, have been introduced in Refs. [2,4] and constitute an essential ingredient to reproduce the large amount of data in the $f_{7 / 2}$ shell. The $V_{C m}$ term takes into account the Coulomb effects due to changes in the nuclear radius as a function of the angular momentum. Radii are strictly dependent on the occupation of the valence orbitals. In the $f p$ shell this term was parametrized by considering that changes of the radius depend on the variation of the occupation of the $p$ orbitals, which have larger radii than the $f$ ones [4]. This has been recently discussed in detail in Ref. [1]. Indeed, the effect is still larger in the $s d$ shell between the $s$ and the $d$ orbits $[1,15]$. In describing the MED for $A=23$ we thus estimate this term from the variation of the average of the occupation number $m=(z+n) / 2$ of protons and neutrons of the $s_{1 / 2}$ orbit with respect to the ground state (g.s.): $\Delta\left\langle V_{C m}\right\rangle_{J}=$ $a_{r}\left[m_{s_{1 / 2}}\right.$ (g.s.) $\left.-m_{s_{1 / 2}}(J)\right]$, with $a_{r}=200 \mathrm{keV}$, the same value used for the $f_{7 / 2}$ shell [3].

The $V_{B}$ term has been deduced in Refs. [2,3] from the MED data of the $A=42$ mirrors and revisited in a recent work by Bentley et al. [16]. We adopt a similar parametrization: $\Delta\left\langle V_{B}\right\rangle_{J}=-100 \mathrm{keV}\left(\left\langle V_{\pi \pi}^{I=0}\right\rangle_{J}-\left\langle V_{\nu \nu}^{I=0}\right\rangle_{J}\right)$, where the $V_{i i}$ operators have just 3 matrix elements different from zero with unit value: those of two identical nucleons in the same orbit coupled to $I=0$. Here, at variance with the nuclei in the $f_{7 / 2}$ where this orbital dominates the wave functions, we consider the three $s d$ orbitals on the same footing.

Calculations have been performed with the code ANTOINE [17]. The contributions of the different terms to the MED are reported in Fig. 3. The trend of the experimental MED is well reproduced by the Coulomb multipole term $V_{\mathrm{CM}}$. However, it is clear that the radial term $V_{C m}$ and the ISB term $V_{B}$ are needed to better reproduce the experimental data, excluding the $15 / 2^{+}$state where they

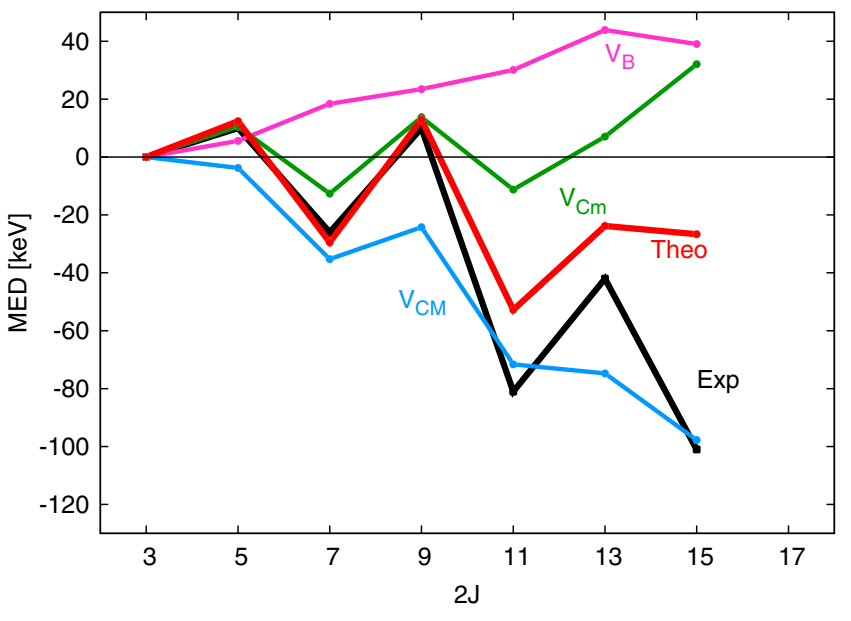

FIG. 3. Experimental MED compared with the theoretical ones obtained with the USD interaction. The ISB part $V_{B}$, the radial contribution $V_{C m}$, and the multipole term $V_{\mathrm{CM}}$ are enlightened.

worsen the fit. When trying a similar description of other MED in the $s d$ shell, although data are limited to lower spins, the inclusion of these two terms with the same parametrization seems to improve the description of MED data [18]. We can therefore conclude that both the radial $V_{C m}$ and the ISB $V_{B}$ terms deduced for the $f_{7 / 2}$ shell are not confined to this orbital but constitute a more general feature.

The second approach we follow here is inspired by the recent theoretical work by Bonnard et al. [1], where mirror nuclei consisting on a closed-shell core plus a particle or a hole have been studied. By adopting the charge-dependent effective interaction (MCI) [15], derived from the chiral N3LO realistic nucleon-nucleon potential [19] in a no-core approach, all electromagnetic and nuclear ISB contributions are naturally included. Two important mechanisms have been put in evidence by Bonnard and collaborators in Ref. [1]: (i) when a particle is added to a system it drives the radii of neutrons and protons in different directions, tending to equalize the radii of both fluids independently of the neutron excess [isovector monopole polarizability (IMP)]; and (ii) the low- $l$ orbits in a main shell have a much larger radius than the rest. These two mechanisms are at the basis of the radial term $V_{C m}$ empirically adopted above. Indeed, we have accounted for the change in radius by considering the variation of (i) the average number of protons and neutrons in the (ii) $s$ orbit. Still there is an important ingredient missing in that approach: the nuclear skin. Indeed, while the IMP effect tends to reduce the skin, its value is not at all vanishing and we will show that the MED can give an insight into the nuclear skin and its evolution as a function of the angular momentum.

We apply here, for the first time, the approach introduced for one valence nucleon in Ref. [1] to deformed nuclei where several valence particles are active and interact with 
each other. The isovector monopole polarizability is here taken into account by calculating the matrix elements of the MCI interaction in the harmonic oscillator basis considering a different size parameter for protons and neutrons in both mirror partners. The size parameters $\hbar \omega_{\pi, \nu}$ are inversely proportional to the respective radii $\rho_{\pi, \nu}[1,14]$. Now, the proton radius $\rho_{\pi<}$ of the $Z<N$ nucleus ${ }^{23} \mathrm{Na}$ is known experimentally and isospin symmetry implies that it is the same as the neutron radius $\rho_{\nu>}$ of its $Z>N$ mirror ${ }^{23} \mathrm{Mg}$. On the other hand, the proton radius $\rho_{\pi>}$ of ${ }^{23} \mathrm{Mg}$, that is not known, is deduced from the difference in binding energy of both nuclei, the so-called mirror displacement energy (MDE), which is measured [20]. By isospin conservation, it is considered equal to $\rho_{\nu<}$ [1]. This is the basic idea behind the work of Duflo and Zuker [14] who fitted the experimentally known mean square proton radii with the formula

$$
\rho_{\pi}=A^{1 / 3}\left[\rho_{0}-\frac{\zeta}{2} \frac{t_{z}}{A^{4 / 3}}-\frac{v}{2}\left(\frac{t_{z}}{2}\right)^{2}\right] e^{g / A}+\lambda \mathcal{D}_{\nu \pi},
$$

where $t_{z}=N-Z$. The coefficients $\rho_{0}, \zeta$, and $v$ are associated with the scalar, vector, and tensor components of $\rho_{\pi}$, while the $e^{g / A}$ factor corrects for the larger radii observed in light nuclei. The expression of the correction term $\mathcal{D}_{\nu \pi}$ may be found in Refs. [1,14,15] where its efficiency in providing very good radii and its physical interpretation in terms of correlations are also discussed.

The vector term parametrized by $\zeta$ is strictly related to the difference of neutron and proton radii, the neutron skin:

$$
\Delta r_{\nu \pi}=\rho_{\nu}-\rho_{\pi}=\frac{\zeta t_{z}}{A} e^{g / A} .
$$

Therefore, by fitting charge radii, the neutron radius can be deduced from $\zeta$. It has been shown that the quality of the 5 -parameters fit of all charge radii of nuclei with mass $A<60$ and $N>Z$ using Eq. (1) turns out to be independent from the value of $\zeta$ as far as it is within a reasonable range of $0.3-1.2$ [1]. This offers the possibility to deduce the value of the neutron skin and, therefore, of the neutron radius $\rho_{\nu}$ in order to reproduce the MDE, keeping the fit of all charge radii in very good agreement with data. In the same way, for each pair of analog excited states, the optimal value of $\zeta$ that allows us to reproduce the experimental MED can be determined.

The trend of the calculated MED with respect to the variation of $\zeta$ is reported in Fig. 4 for $A=23$. Noticeably, the variation of $\zeta$ implies a shift in all the MED but does not change the relative values for the different $J$ states: The calculated MED follow a linear trend, decreasing as $\zeta$ increases. This linear trend can be exploited to determine the optimal value of $\zeta$, and hence of the neutron skin, needed to reproduce the experimental MED value for each level. The resulting values are reported in Table I.

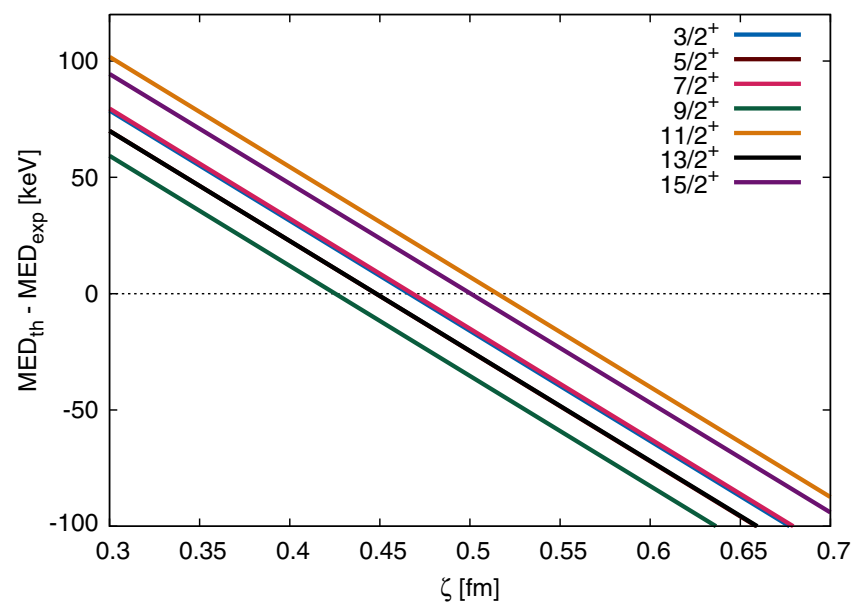

FIG. 4. Calculated MED for the $A=23$ mirror pair as a function of the $\zeta$ parameter.

Variations up to $20 \%$ in the skin are needed to reproduce the data. The experimental information on the nuclear size is generally confined to the ground state charge radii. This result is therefore of remarkable importance since it allows us to estimate the neutron skin for each excited state from the experimentally measured MED values.

To interpret this state-dependent behavior we recall the relation between the variation of the nuclear radius with the occupation of the $s_{1 / 2}$ orbital. Therefore, we look for a correlation of the neutron skin with the difference in occupation numbers of neutrons and protons of the $s_{1 / 2}$ orbit as a function of $J, \Delta_{\nu \pi}(J)$. We report in Fig. 5(a) the values of $\zeta$ and $\Delta_{\nu \pi}$ for ${ }^{23} \mathrm{Na}$. The correlation is striking.

We have performed the same analysis for other $T=1 / 2$ mirror pairs in the $s d$ shell. The results, confirming the correlation between $\zeta$ and $\Delta_{\nu \pi}$, are shown in Fig. 5(b).

It is worth considering the physical meaning of this result. In the "usual" approach adopted in the systematic studies of the $f_{7 / 2}$ shell the radial term is related to the average occupation number of low- $l$ orbit of protons and neutrons [2,3]. It gives therefore an isoscalar contribution to the MED. In addition to a realistic treatment of these effects, the new generalized approach presented here also incorporates an isovector contribution to the MED, correlated to the neutron skin, through the difference between neutron and proton occupations.

It is evident from Fig. 5 how the trend of $\Delta_{\nu \pi}$ is in phase with $\zeta$ except for the case of the $A=23$ mirror nuclei. The origin of this effect is not clear and further investigations are ongoing.

TABLE I. Deduced neutron skin $\Delta r_{\nu \pi}$ (fm) in ${ }^{23} \mathrm{Na}$ for each yrast excited state.

\begin{tabular}{lccccccc}
\hline \hline$J$ & $3 / 2$ & $5 / 2$ & $7 / 2$ & $9 / 2$ & $11 / 2$ & $13 / 2$ & $15 / 2$ \\
\hline$\Delta r_{\nu \pi}$ & 0.0211 & 0.0202 & 0.0211 & 0.0192 & 0.0233 & 0.0202 & 0.0226 \\
\hline \hline
\end{tabular}



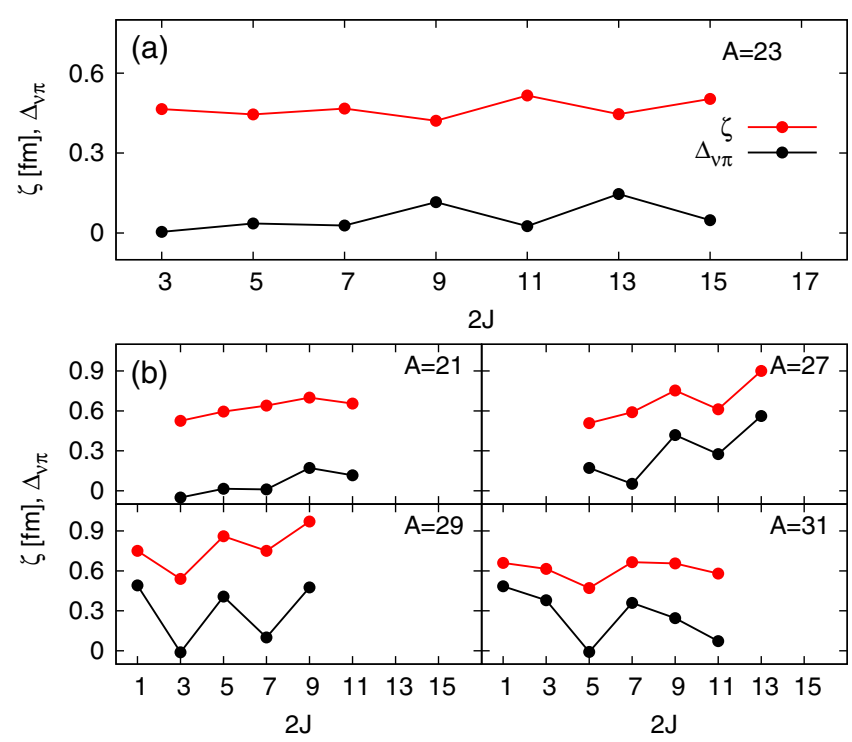

FIG. 5. The parameter $\zeta$ and the difference in the occupation number of the $s_{1 / 2}$ orbital between neutrons and protons: (a) for the mirror nuclei $A=23$; (b) for some $T=1 / 2$ mirror nuclei in the $s d$ shell.

In summary, the level scheme of ${ }^{23} \mathrm{Mg}$ has been extended to high spin and compared to its mirror ${ }^{23} \mathrm{Na}$, populated in the same experiment. This has allowed us to study the behavior of the MED along the rotational bands through several states up to the $15 / 2^{+}$for the first time in the $s d$ shell. The MED have been reproduced with good accuracy by means of shell model calculations using the method successfully developed for $f_{7 / 2}$-shell nuclei, here extended to the $s d$. The important role of the schematic radial $V_{C m}$ and the ISB $V_{B}$ terms has been put in evidence. A novel approach based on the use of a charge-dependent realistic effective interaction and taking into account the effect of the isovector monopole polarizability has allowed us to deduce from the MED, for the first time, the neutron skin for each excited state. A striking correlation between the difference in the occupation numbers of neutrons and protons in the $s_{1 / 2}$ orbital and the neutron skin has been found, giving insight into the isovector contribution to the radial term of the MED.

The next goal is to find the quantitative relation between the difference in the occupation numbers of neutrons and protons of the $s_{1 / 2}$ orbit and the skin value. This will allow us to deduce the latter from the former and to naturally include the isovector polarizability in the interaction matrix elements used in shell-model calculations. The predictive capability of such an approach will be extremely powerful.

The authors are grateful to the GANIL accelerator team. This work has been financed by the European Union Seventh Framework Program No. FP7/2007-2013 under Grant Agreement No. 262010 ENSAR.

*Present address: National Physical Laboratory-Hampton Road, Teddington, Middlesex, TW11 0LW, United Kingdom.

alberto.boso@npl.co.uk

†Present address: Institut für Kernphysik, Technische Universität Darmstadt, 64289 Darmstadt, Germany.

[1] J. Bonnard, S. M. Lenzi, and A. P. Zuker, Phys. Rev. Lett. 116, 212501 (2016).

[2] A. P. Zuker, S. M. Lenzi, G. Martinez-Pinedo, and A. Poves, Phys. Rev. Lett. 89, 142502 (2002).

[3] M. A. Bentley and S. M. Lenzi, Prog. Part. Nucl. Phys. 59, 497 (2007).

[4] S. M. Lenzi et al., Phys. Rev. Lett. 87, 122501 (2001).

[5] A. Gadea et al., Phys. Rev. Lett. 97, 152501 (2006).

[6] D. G. Jenkins et al., Phys. Rev. C 87, 064301 (2013).

[7] J. Gomez del Campo, D. E. Gustafson, R. L. Robinson, P. H. Stelson, P. D. Miller, J. K. Bair, and J. B. McGrory, Phys. Rev. C 12, 1247 (1975).

[8] D. Evers et al., Z. Phys. A 280, 287 (1977).

[9] J. Scheurer et al. Nucl. Instrum. Methods Phys. Res., Sect. A 385, 501 (1997).

[10] Ö Skeppstedt et al., Nucl. Instrum. Methods Phys. Res., Sect. A 421, 531 (1999).

[11] F. Azaiez, Nucl. Phys. A654, 1003c (1999); J. Simpson et al., Heavy Ions Phys. 11, 159 (2000).

[12] B. A. Brown and B. H. Wildenthal, Annu. Rev. Nucl. Part. Sci. 38, 29 (1988).

[13] J. A. Nolen and J. P. Schiffer, Annu. Rev. Nucl. Sci. 19, 471 (1969).

[14] J. Duflo and A. P. Zuker, Phys. Rev. C 66, 051304 (2002).

[15] J. Bonnard and A. P. Zuker, J. Phys. Conf. Ser. 1023, 012016 (2018).

[16] M. A. Bentley, S. M. Lenzi, S. A. Simpson, and C. A. Diget, Phys. Rev. C 92, 024310 (2015).

[17] E. Caurier and F. Nowacki, Acta Phys. Pol. B 30, 705 (1999).

[18] S. M. Lenzi and R. Lau, J. Phys. Conf. Ser. 580, 012028 (2015).

[19] D. R. Entem and R. Machleidt, Phys. Lett. B 524, 93 (2002).

[20] M. Wang, G. Audi, A. H. Wapstra, F. G. Kondev, M. MacCormick, X. Xu, and B. Pfeiffer, Chin. Phys. C 36, 1603 (2012). 